\title{
AVALIAÇÃO DO USO CONSCIENTE DAS PLANTAS MEDICINAIS POR FREQUENTADORES DE UMA UNIDADE BÁSICA DE SAÚDE DE PORANGATU-GO
}

Elaine Gomes da SILVA ${ }^{1}$

Débora Cristina da Silva LIMA ${ }^{2}$

Camila Regina do VALE ${ }^{2}$

\begin{abstract}
${ }^{1}$ Pós-graduanda do curso de Especialização em Tecnologias Aplicadas ao Ensino de Biologia (ETAEB) do Instituto de Ciências Biológicas da Universidade Federal de Goiás.

${ }^{2}$ Doutorandas em Ciências Biológicas - área de concentração: Genética e Bioquímica pela UFG. camilarvale@ hotmail.com.
\end{abstract}

Recebido em: 10/05/2016 - Aprovado em: 28/10/2016 - Disponibilizado em: 18/12/2016

\begin{abstract}
RESUMO:
$\mathrm{O}$ uso derecursos disponíveis na natureza foi à primeira forma de tratamento para algumas doenças em períodos mais remotos, desde então o homem passou a selecionar espécies eficazes para combater sintomas e doenças. O uso de plantas medicinais pela população brasileira é uma prática tradicional, sendo muitas vezes o único recurso utilizado na atenção básica de saúde. Este trabalho objetivou fazer um levantamento etnobotânico das plantas medicinais mais utilizadas por frequentadores de uma Unidade Básica de Saúde (UBS) no município de Porangatu/GO e avaliar o uso destas espécies pela sua comunidade. Neste levantamento foi apontado que todos os entrevistados de um total de 40 pessoas utilizamplantas medicinais para alívio de sintomas indesejados, as formas de consumo em sua maioria foi o chá (80\%). Foi observado que o conhecimento adquirido sobre a utilização das plantas é predominantemente familiar (95\%). Do total de 40 entrevistados, 20 (50\%) cultivam as plantas em quintais, apenas 14 (35\%) conhecem e citaram algum tipo de planta tóxica. Em relação à frequência de uso 77,5\% dos entrevistados disseram utilizar raramente de remédios feitos de plantas medicinais.Os dados obtidos permitem constatar que é abrangente a utilização das plantas medicinais no município de Porangatu-Go comparando aos entrevistados da UBS, observando também um conhecimento precário sobre plantas tóxicas, ressaltando a importância de programas voltados para a orientação e esclarecimento das práticas de utilização das plantas medicinais em UBS's as quais são frequentadas assiduamente por pessoas do bairro a fim de evitar casos de intoxicações e efeitos colaterais.

Palavras-chave: Plantas medicinais. Toxicidade. Saúde. Etnobotânica. Unidade Básica de Saúde.
\end{abstract}

\section{ABSTRACT:}

The use of resources available in nature was the first form of treatment for some diseases in earlier periods, since man began to select efficient species to combat symptoms and diseases. The use of medicinal plants by the Brazilian population is a traditional practice, often the only resource used in basic health care. This study aimed to do an ethnobotanical survey of medicinal plants commonly used by patrons of a Basic Health Unit (BHU) in the city of Porangatu / GO and evaluate the use of these species for their community. In this survey it was noted that all respondents from a total of 40 people use medicinal plants to relieve unwanted symptoms, forms of consumption mostly was the tea $(80 \%)$. It was observed that the knowledge gained about the use of the plant family is predominantly (95\%). Of the 40 respondents, $20(50 \%)$ cultivate the plants in gardens, only $14(35 \%)$ know and cited some sort of toxic plant. Regarding the frequency of use $77.5 \%$ of respondents said they rarely use made of herbal remedies. The data obtained allow us to state that it is comprehensive use of medicinal plants in Porangatu-Go municipality compared to UBS respondents also observing a poor knowledge of toxic plants, highlighting the importance of programs for guidance and clarification of the use of practices medicinal plants in UBS's which are frequented assiduously by people in the neighborhood in order to prevent cases of poisoning and side effects.

Keywords: Medicinal plants. Toxicity. Health. Ethnobotany. Basic health Unit.

\section{INTRODUÇÃO}

A utilização de plantas medicinais é uma prática comum e bastante difundida entre as populações, especialmente, as de cidades interioranas em que se pode notar forte influência da cultura da medicina popular 
(SILVA et al., 2012). Sendo considerada uma alternativa importante para muitas populações de baixo poder aquisitivo já que contribuem para redução dos gastos com medicamentos sintéticos (SILVA e SOUZA., 2007).

Pode-se considerar como planta medicinal aquela administrada sob qualquer forma e por alguma via ao homem, exercendo algum tipo de ação farmacológica. As plantas podem ser classificadas de acordo com sua ordem de importância iniciando pelas plantas empregadas na terapêutica, seguidas daquelas que constituem matéria-prima para manipulação e, por último, aquelas empregadas na indústria para obtenção de princípios ativos ou como precursores em semi-síntese (ALVES e SILVA., 2003).

Até meados do século XX, as plantas medicinais constituíam a base da terapêutica medicamentosa, quando a síntese química teve início no final do século XIX, iniciou-se uma fase de desenvolvimento vertiginoso. Atualmente cerca de $50 \%$ dos medicamentos utilizados são de origem sintética e cerca de $25 \%$ de origem vegetal, isolados ou produzidos por semi-síntese (VALE.,2002).

Os primeiros registros do uso de fitoterápicos datam da China do período de 3000 a.C. quando o imperador chinês catalogou 365 ervas medicinais e venenos que eram utilizados na época, criando assim o primeiro herbário que se tem notícia(FRANÇA.,2008).No Brasil o uso de plantas medicinais foi herdado das práticas indígenas, influenciada pela cultura africana e portuguesa, tornando-se uma vasta cultura popular (ALVES e SILVA.,2003).

No Brasil diversos estudos já foram realizados com o objetivo de conhecer e divulgar sua flora medicinal, considerando as suas diferentes regiões geográficas e etnias existentes. Vila Verde e colaboradores (2003)realizaram um levantamento etnobotânico com plantas medicinais do cerrado, utilizadas pela população de Mossâmedes em Goiás e chamaram a atenção para o fato da população fazer o uso de plantas medicinais por indicação de raizeiros, que desenvolvem o extrativismo e comercializam em pequena escala as plantas nativas da região. Trabalho semelhante foi realizado por Rizzo e colaboradores (1999), onde foi avaliado o uso de plantas medicinais nas cidades de Goiás e Pirenópolis. Igualmente Costa Neto e Oliveira (2000) pesquisaram também o uso de plantas medicinais na cidade de Taquinho no Estado da Bahia.

Estudos da medicina popular vêm merecendo atenção cada vez maior devido ao contigente de informações e esclarecimentos oferecidos à ciência. Esse fenômeno tem propiciado o uso de chás, decoctos, tisanas e tinturas fazendo com que, na maioria dos países ocidentais, os medicamentos de origem vegetal sejam retomados de maneira sistemática na profilaxia e tratamento de doenças, ao lado da terapêutica convencional 
(VALE.,2002).As plantas medicinais têm grande influência na manutenção das condições de saúde de pessoas. Isso se deve em parte ao aumento dos estudos com fitoterápicos, levando a uma comprovação da ação terapêutica de várias plantas utilizadas popularmente, mas, principalmente, ao fato de que a fitoterapia é parte da cultura da população sendo utilizada e difundia há muitas gerações(KLEIN et al., 2009).

O Ministério da Saúde (MS) aprovou em 2006 a Política Nacional de Plantas Medicinais e Fitoterápicos por meio do decreto 5.813, de 22 de Junho de 2006, justificada pela presença da ciência fitoterápica no cotidiano das pessoas, aliado ao fato de o Brasil ser o país que detém a maior parcela da biodiversidade mundial, em torno de 15 a 20\% (BRASIL, 2006).

Ações políticas têm como por objetivo promover o uso sustentável dos componentes da biodiversidade brasileira, conduzindo a geração de riquezas com inclusão social e melhoria na qualidade de vida. Objetiva-se também ampliar as opções terapêuticas e melhorar a atenção a saúde dos usuários do Sistema Único de Saúde (SUS), sem deixar de valorizar e preservar o conhecimento das comunidades e povos tradicionais(BRASIL, 2009).

Diversas espécies de plantas são utilizadas na terapêutica de diferentes enfermidades e seu uso pode variar de uma região para outra.Estudos realizados em um bairro rural do Município de Varjea GrandeSPdemonstra uma variedade de plantas citadas pelos moradores para o tratamento de várias doenças. Como por exemplo a Brassica olareacea (couve) e a Baccharis sp para diminuir as taxas de açúcar no sangue, a Lactuta sativa L. (alface) para tratar da pressão alta, a Chenopodium ambrosioides L.para tratarcistite e algia de bexiga, o Coronopus didymus L. Sm. (mentruz) para curar lesões da pele, entre outras (SANTOS et al., 2008).

Apesar do uso de plantas medicinais para tratamento, cura e prevenção de determinadas doenças ser uma das mais antigas formas de prática medicinal da humanidade e estar apoiada a um conhecimento consolidado por séculos de observação, planta medicinal não é sinônimo de inocuidade. Ao contrário do senso comum de que "medicamento natural não fizer bem, mal não faz", planta medicinal é um xenobiótico, ou seja, um produto estranho ao organismo com finalidades terapêuticas, que ao ser introduzido no organismo humano sofre biotransformação e pode, desta forma gerar produtos tóxicos (VEIGA JUNIOR et al., 2005; NICOLETTI et al., 2007).

Em muitos casos as pessoas subestimam as propriedades medicinais das plantas e fazem o uso delas de forma aleatória. Entretanto, cada vegetal em sua essência, pode ser alimento, veneno ou medicamento. A distinção entre as substâncias alimentícias, 
tóxicas e medicamentosas se faz apenas com relação a dose, a via de administração e a finalidade com são empregadas (MORGAN., 1982). A sociedade tem a percepção de que todo produto natural é seguro e desprovido de efeitos colaterais, em alguns casos os efeitos dos produtos naturais são apenas psicológicos e, em outros, causam danos irreversíveis à saúde (NEWMANeGRAGG., 2007).

Os efeitos mais preocupantes do uso indiscriminado de plantas medicinais são teratogênico, embriotóxico e abortivo, uma vez que os constituintes da planta podem atravessar a placenta e gerar um desses efeitos (BRASIL., 2005). Para Embiruçui e colaboradores (2005) os teratógenos constituem agentes ambientais, químicos, físicos e biológicos que podem causar anormalidades obstetra e/ou fetais. Algumas pesquisas demostraram que muitas dessas plantas possuem substâncias agressivas e por essa razão devem ser utilizadas com cuidado respeitando seus riscos toxicológicos (VEIGA JUNIOR et al, 2005).

As plantas são organismos complexos, e como tais, apresentam um metabolismo extraordinário, que leva a produção de uma grande variedade de substâncias químicas, dentre substâncias que podem ser tóxicas e irritantes para alguns organismos. No entanto a simples presença dessas substâncias em uma determinada espécie vegetal não é suficiente para qualifica-la como tóxica (OLIVEIRA et al., 2003).O conhecimento dos componentes químicos das plantas é fundamental no esclarecimento dos diferentes aspectos relacionados aos casos de intoxicação e para identificação de potenciais substâncias químicas de ação tóxica (SOUZA et al., 2010).

As intoxicações ocorrem quase sempre razão do uso de quantidades excessivas de determinados vegetais, do preparo e uso inadequados, e principalmente em virtude do uso de espécies com efeitos tóxicos (MARTINS et al., 1998).Segundo Pinillose colaboradores (2003), as intoxicações por plantas são frequentes tanto pelo consumo de espécies tóxicas por erro de identificação, quanto pelo excesso na quantidade ingerida, sem falar no consumo negligente das crianças quando ingerem partes de plantas coloridas e atrativas durante as brincadeiras. Também é bastante comum atendimento de urgência sem a associação dos sintomas com o produto ingerido, o que dificulta o diagnóstico etiológico.

Futuro e colaboradores enfatizam o fato que vários outros problemas contribuem para ocorrência de interação entre planta e medicamentos, bem como pela baixa difusão desta informação na sociedade. Entre estes fatores destacam-se: os problemas de identificação das espécies vegetais, escassez de estudos clínicos sobre interações medicamentosas e a falta de incorporação das plantas nos programas de farmacovigilância. 
Outra questão a se considerar é que, infelizmente, as informações técnicas e científicas geradas dentro das universidades nem sempre chegam à população. Por isso cabe aos pesquisadores divulgarem as indicações terapêuticas, toxicidade, contra indicações e possíveis interações entre plantas e medicamentos e orientar a população quanto ao uso correto das plantas medicinais/fitoterápicos, evitando seu uso indiscriminado. É necessária uma interação cada vez maior da universidade com a comunidade para que a população seja informada dos benefícios e riscos da fitoterapia. (BRITTO et al.,2008).

O presente trabalho objetivou avaliar o uso consciente das plantas medicinais no município de Porangatu-Go, levantando informações referentes ao uso, ao cultivo, e formas de utilização das plantas medicinais, além de verificar o grau de conhecimento do público sobre a identificação de plantas tóxicas, investigando relatos sobre os efeitos adversos que os entrevistados sentiram ao fazer uso de plantas medicinais.

\section{MATERIAL E MÉTODOS}

\section{1. Área de estudo}

O presente estudo foi realizado em uma Unidade Básica de Saúde (UBS) do Município de Porangatu- Go. Localizado a 420 quilômetros de Goiânia-Go.

\subsection{Sujeitos da pesquisa}

A pesquisa foi realizada com a participação de 40 frequentadores da UBS entre pacientes e acompanhantes.

\subsection{Coleta e análise dos dados}

O presente trabalho foi desenvolvimento em apenas uma etapa, onde os frequentadores foram abordados pela entrevistadora e esclarecidos sobre o objetivo da pesquisa e da importância da participação deles e que após expor a relevância do trabalho pediu a colaboração voluntária dos mesmos. Aqueles que consentiram em participar da pesquisa assinaram um Termo de consentimento de participação e responderam um questionáriosobre dados sócio - demográficos, econômicos e sobre o uso de plantas medicinais. Os dados foram mensurados em números absolutos e percentuais, sendo que os resultados das questões abertas foram analisados de forma descritiva.

\section{RESULTADOS}

Foram aplicados 40 questionários respondidos pelos frequentadores da UBS, ondetodos os participantes eram do Município de Porangatu, sendo que97,5\% eram da zona urbana e apenas um 2,5\% era da zona rural. Sendo que $90 \%$ eram do sexo feminino e $10 \%$ do sexo masculino representados no Figura 1. 


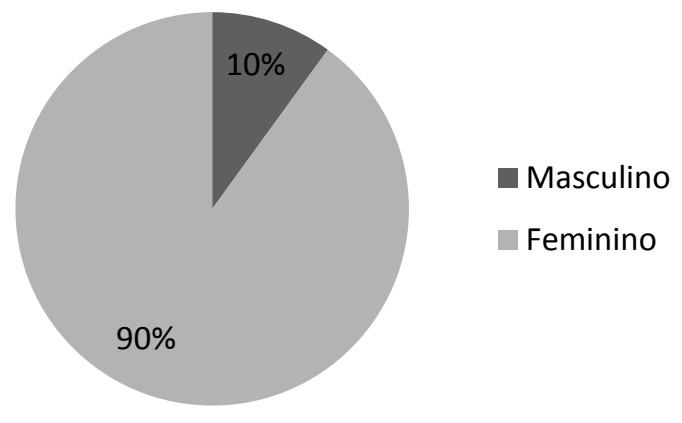

Figura 1: Percentual do sexo dos entrevistados.

Em relação á idade $52 \%$ estava acima de 40 anos, e $48 \%$ entre 18 e quarenta anos, descritos no Figura 2 Os participantes apresentaram grau de escolaridade variado, naFigura 3estão apresentados os graus de escolaridade, com respectivos valores percentuais dos colaboradores que participaram da pesquisa.

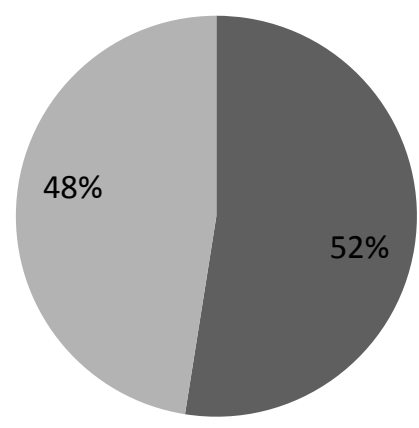

Acima de 40 anos

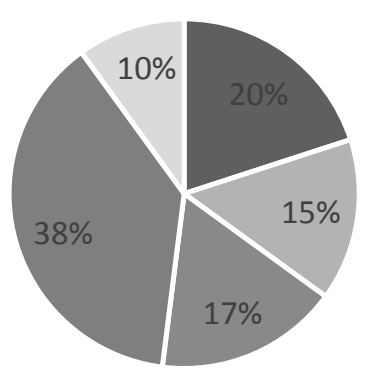

- Não

alfabetizado

- Nível

fundamental I

- Nível

fundamental II

- Nível médio

Ensino

superios

Figura 3: Dados sobre a escolaridade dos entrevistados.

Quando perguntados se fazem uso de plantas medicinais todos responderam que sim. Sendo que $80 \%$ consumem em forma de chá, $10 \%$ em forma de suco e $10 \%$ em forma de garrafada e chá, descritos no Figura 4.

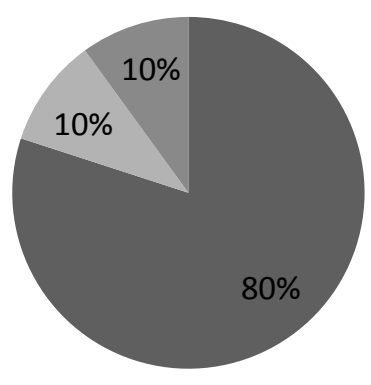

u consome em forma de chá

forma de suco

forma de garrafada e chá

Figura 4: Forma de consumo as plantas medicinais. 
Dos entrevistados (97\%) participantes disseram conhecer algum tipo de planta medicinal, e um (2\%) disse não conhecer.

As principais plantas citadas pelos entrevistados estão na Tabela 1 apresentadas. Foram citadas principalmente as seguintes plantas: o Coleus amboinicus Lour (hortelã gorda), o Macrosyphonia velame (velame branco), o Gossypium hirsutum (algodão), o Chenopodium ambrosoides (Matruz), a Peumus boldus Molina (boldo do chile), a Aloe vera L.(babosa), o Mentha pulegium (poejo) $e$ a Melissa officinalis (erva cidreira).

Tabela 1: Relação das plantas medicinais utilizadas pela comunidade

\begin{tabular}{lll}
\hline Nome científico & Nome popular & Família \\
\hline $\begin{array}{l}\text { Coleus amboinicus } \\
\text { Lour }\end{array}$ & Hortelã gorda & Lamiaceae \\
Macrosyphonia velame & Velame branco & Apocinaceae \\
Gossypium hirsutum & Algodão & Malvaceae \\
Chenopodium & Mastruz & \\
ambrosoides & & Brassicaceae \\
Peumus boldus Molina & Boldo do chile & Monimiaceae \\
Aloe vera l. & Babosa & Liliaceae \\
Mentha pulegium & Poejo & Laminaceae \\
Melissa officinais & Erva cidreira & Laminaceae \\
\hline
\end{tabular}

Em relação ao conhecimento sobre plantas medicinais, $85 \%$ disseram que o conhecimento era de origem familiar, 5\% disseram ter adquirido o conhecimento através das mídias e $10 \%$ tinha conhecimento familiar e através de contato com profissionais da saúde conforme o Figura 5.

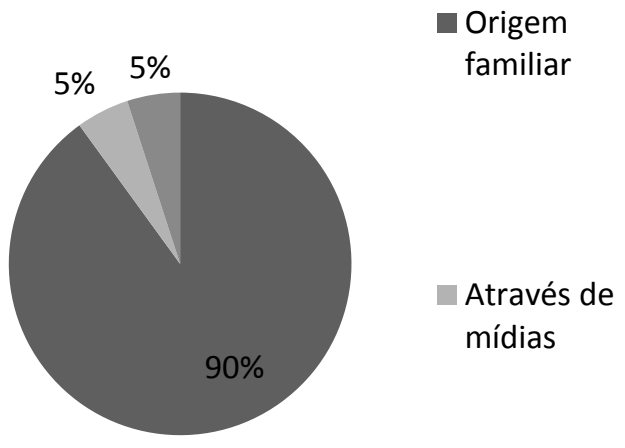

Figura 5: Origem do conhecimento sobre uso de plantas medicinais.

Quanto à aquisição das plantas medicinais,53\% disseram ser através do cultivo próprio, $18 \%$ através de vizinhos, $5 \%$ cultivo próprio e vizinhos, um $3 \%$ cultivo próprio e compra em feiras, um 3\% de outras formas (busca em matas) e 18\% compra em feiras. Os dados estão descritos no Figura6.

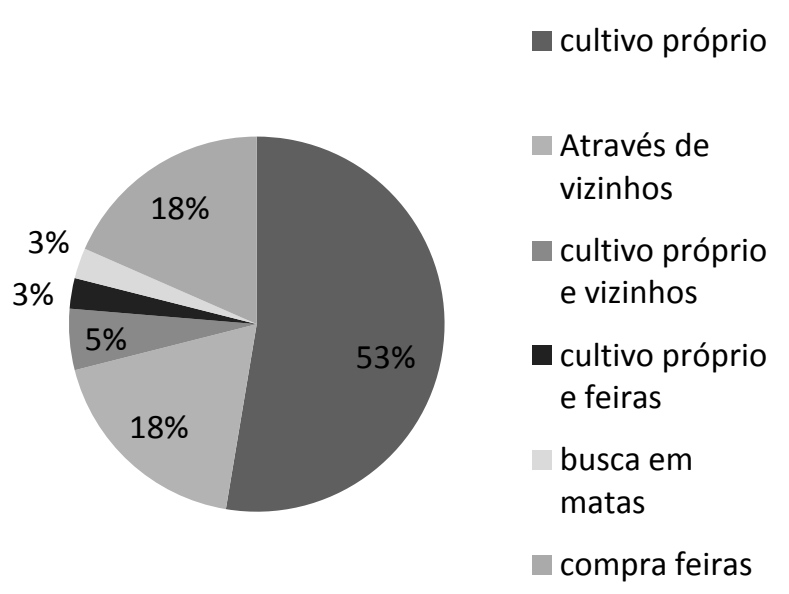

Figura 6: Formas de aquisição.

Em relação à frequência de consumo $77 \%$ dos entrevistados disseram fazer uso 
raramente, $18 \%$ disseram fazer uso uma vez por semana e apenas $5 \%$ fazem uso todo dia (Figura 7).

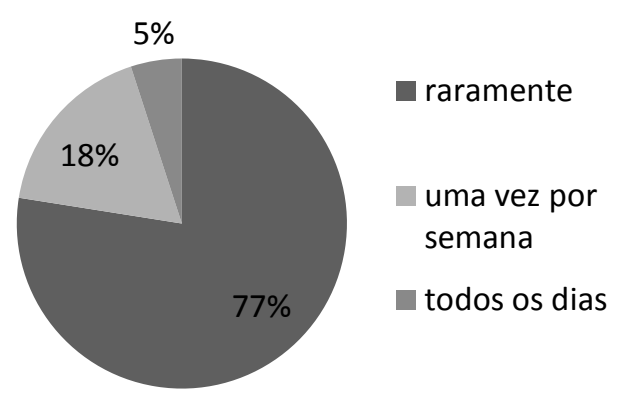

Figura 7: Frequência de consumo.

Ao serem perguntados se já sentiram mal ao fazer uso de plantas medicinais 39 (98\%) disseram que não e um (2\%) disse que sim, ao consumir chá da folha do mamão (Carica papaya L.), os dados estão descritos na Figura 8.

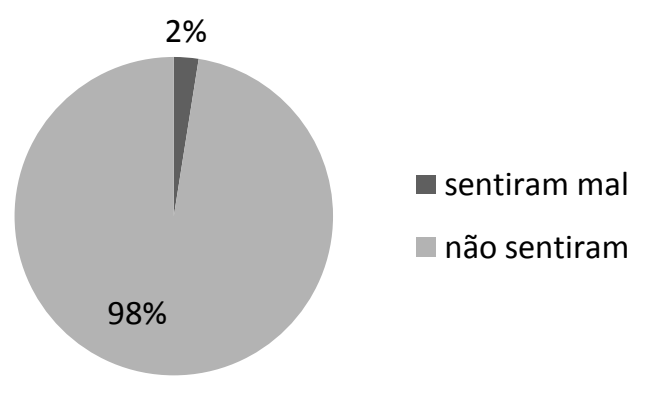

Figura 8: Entrevistados que sentiram mal ao fazer uso das plantas medicinais.

Em relação ao conhecimento sobre plantas tóxicas, 26 (65\%) disseram conhecer algum tipo de planta tóxica 14 (35\%) disseram não conhecer. $\mathrm{Na}$ Figura 9 estão descritos os resultados absolutos dos que conhecem, na tabela 02 estão descritas as plantas citadas como tóxicas.

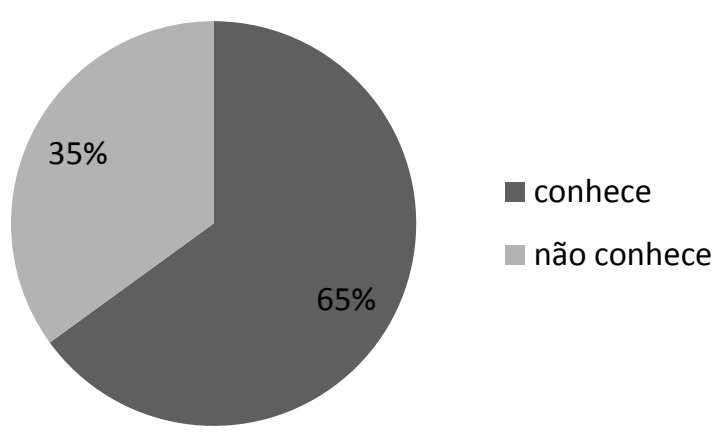

Figura 9: Entrevistados que conhecem plantas tóxicas.

Tabela 2: Relação das plantas citadas como tóxicas

\begin{tabular}{ccc}
\hline Nome científico & $\begin{array}{c}\text { Nome } \\
\text { popular }\end{array}$ & Família \\
\hline Ruta graveolens & Arruda & Rutaceae \\
Aloe vera & Babosa & Liliaceae \\
Petivera alliceae L. & Tipi & Phytolaccaceae \\
Luffa operculata & Buchinha & Curcubitaceae \\
Atropa beladona & Beladona & Solanaceae \\
\hline
\end{tabular}

\section{DISCUSSÃO}

O presente trabalho teve como objetivo avaliar o nível de conhecimento dos 
frequentadores de uma UBS sobre plantas medicinais e suas formas de utilização. Uma vez que as plantas é uma alternativa medicinal de baixo custo e na maioria das vezes o único recurso disponível para a população estudada.

As plantas medicinais tem grande influência na manutenção das condições de saúde das pessoas. Isso se deve em parte ao aumento dos estudos com fitoterápicos, levando a uma comprovação da ação terapêutica de várias espécies utilizadas popularmente, mas principalmente, ao fato de que a fitoterapia é parte da cultura da população, sendo utilizada e difundida há muitas gerações (KLEIN et al.,2009).

Estudos realizados em Nazaré PaulistaSP Hoeffel e colaboradores (2006) demonstram que o conhecimento acerca de plantas medicinais está relacionado essencialmente ao sexo feminino,fato esse que foi confirmado no presente estudo onde a maioria dos entrevistados que afirmaram usar plantas medicinais era do sexo feminino.

No presente estudo, em relação ao uso de plantas medicinais, todos disseram fazer. A idade dos participantes influenciou, sendo que aqueles acima de 40 anos, souberam citar maior número de espéciesmedicinais e aquelas que consideraram como tóxicas. $\mathrm{O}$ trabalho realizado nas Áreas de Proteção Ambiental (APA'S)de Cantareiras/SP e Fernão Dias/MGpor Hoeffel e colaboradores (2006)corrobora com esse resultado obtido no presente estudo, ondefoi observado que os jovens têm pouco interesse em aprender as práticas sobre a utilização das plantas medicinais.

Observou-se também um grau de escolaridade variado, onde apenas 8 participantes não eram alfabetizados, e que esse dado também não teve influência no conhecimento sobre o uso das plantas medicinais. Resultados similares foram obtidos por Brasileiro et al. (2008).

$\mathrm{O}$ conhecimento dos participantes em sua maioria é oriundo da família. Esse dado é confirmado pelos resultados obtidos no município de Nazaré Paulista-SP Hoeffel et al. (2006). Porém vale ressaltar que alguns citaram ter indicação e esclarecimento profissionais de saúde, assim como resultados obtidos na Região Centro-Norte do Estado do Rio de Janeiro por Veiga Júnior (2008),salientando a importância desses profissionais para a valorização da cultura e práticas do uso consciente das plantas medicinais.

O cultivo próprio é a forma predominante de aquisição das plantas medicinais pelos entrevistados. Resultados similares foram demonstrados por Hoeffel et al. (2006) em duas comunidades de Nazaré Paulista- SPe Camanducaia/MG, onde a maioria dos entrevistados também utilizam as espécies que são encontradas em matas.

A forma de consumo mais descrita foi o chá, sendo confirmado em estudos realizados por Brasileiro et al. (2008), 
apontado a preparação em forma de chá como principal meio de utilização das plantas medicinais.

Apenas uma pessoa disse ter sentido mal ao fazer uso de alguma planta medicinal, vale ressaltar que uma proporção considerável dos entrevistados não conhece nenhum tipo de planta tóxica, podendo ocorrer casos de identificação errônea da planta, aumentando os riscos de intoxicação, mesmo os resultados não apontar um número considerável de pessoas que sentiram algum mau depois de utilizar plantas medicinais.Salientando a importância da educação em saúde e no dever do oferecimento desta, pois um dos objetivos do Programa Nacional de Plantas Medicinais e Fitoterápicos é ampliar e inserir a medicina tradicional no Sistema Oficial de Saúde.

\section{CONCLUSÃO}

O uso consciente de plantas medicinais é de fundamental importância para a sociedade, uma vez que deve ser ter conhecimento e esclarecimento adequado para diminuir os riscos de intoxicações e possíveis efeitos colaterais ao longo do tempo. Com base nos resultados obtidos, que demonstram que todos os entrevistados utilizam de alguma planta medicinal, como também em sua maioria souberam citar alguns tipos de plantas que mais utilizam, no entanto não tem um conhecimento abrangente sobre plantas tóxicas, levando-se as seguintes considerações:
- Existe a prevalência total do uso de medicamentos a base de plantas medicinais e o conhecimento popular tradicional ressalva a necessidade de pesquisas que esclarecem e confirmem informações sobre as ações das plantas, objetivando minimizar os riscos de intoxicações e efeitos colaterais, considerando que o seu uso deve ser consciente e seguro.

- É importante o estímulo e a manutenção das práticas relacionadas a utilização das plantas medicinais, considerando que alguns dos entrevistados apesar de relatar que usam plantas medicinais, não conhecem e não souberam citar nenhum tipo. Esses dados apontam que esse fato pode pôr em risco a transferência deste conhecimento para as próximas gerações. Pois o presente trabalho demonstra que o conhecimento é adquirido em sua maioria por familiares.

\section{REFERENCIAS}

\section{ALBUQUERQUE, U. P. Introdução a} etnobotânica. 2. ed. Rio de Janeiro: Interciência, 2005.

ALVES, A.R., SILVA. M.J.P. O uso da fitoterapia no cuidado de crianças com até cinco anos em área central e periférica da cidade de São Paulo: Revista Escola 984 
Enfermagem, USP., v. 37, n.4, p.85-91, 2003.

AMOROZO, M.C., GELY, A. Uso de Plantas Medicinais por Caboclos do Baixo Amazonas. Museu Paraense Emílio Goeldi, Série BotãnicaBelém, v.4, p.47, BarbarenaPA, 1988.

\section{BRASIL. MINISTÉRIO DA}

SAÚDE.Secretaria de Ciência, Tecnologia e Insumos Estratégicos. Departamento de Assistência Farmacêutica e Insumos Estratégicos. Política Nacional de Plantas Medicinais e Fitoterápicos. Brasília: Ministério da Saúde, p. 136- (série C Projetos, Programas e Relatórios, $1^{a}$ ed.) 2006.

BRASIL. MINISTÉRIO DA SAÚDE. Secretaria de Ciência, Tecnologia e Insumos Estratégicos. Departamento de Assistência Farmacêutica e Insumos Estratégicos. Política Nacional de Plantas Medicinais e Fitoterápicos. Brasília: Ministério da Saúde, p. 60- (série B, Textos Básicos de Saúde, 1ª ed.) 2009.

BRASIL. Resolução SES nº 1757, de 18 de Fevereiro de 2002. Contra o uso de Plantas Medicinais no Âmbito do Estado do Rio de Janeiro e dá outras providências. Diário Oficial do Rio de Janeiro, v.2, n.33, Parte 1, 2005.

BRASILEIRO, B.G., PIZZIOLO, V.R., MATOS, D.S., GERMANO, A.M., JAMAL, C.M. Plantas medicinais utilizadas pela população atendida no Programa de Saúde da Família, Governador Valadares - MG, Brasil. Rev. Ciênc. Farm. V. 44. $n^{\circ}$ 4. São Paulo, 2008.

BRITTO, V.L.M.Q., GOUVEIA, N.M., AMARAL, F.C., TEIXEIRA, E.H.M., PEREIRA, W.F., ESPÍNDOLA, F.S. Plantas Medicinais e fitoterápicas no contexto da academia, governo e organizações da sociedade civil: exemplo de inciativas popular no município de Uberlândia- MG. Rev. de Educ. Pop.,v.6, n.1, p. 93-101, 2008.
COSTA - NETO, E.M., OLIVEIRA, M. V.M. The use of Medicinal Plants in the country of Tanquinho, Estates of Bahia Northeasten Brazil. Revista Bras. de Plantas Medicinais. V.2, p.1-8, Botucatu - SP, 2000.

EINSENBERG, D., DAVIS, R.B., ETTNER, S.L. Trends in alternative medicine use the United Estates. Ed. Jama, v.280, n. 18, 1998.

EMBIRUÇU, E.K., SORTE, N.B., VIDA, R., LESSA, L., PANÃO, E., MOTA, A.C., SANTANA, T., ARAÚJO, I., TORALLE, M.B., ACOSTA, A.X. Risco Teratogênico: A percepção em diferentes segmentos da população.Revista de Ciências Médicas e Biológicas., v.4, n. 3, p.201-7, 2005.

FUTURO, D.O., FIORINI, F.S., CASTILHO, S.R. Interações entre plantas e medicamentos. Informativo Ceatrim, n.3, p. 1-4, Niterói RJ, 2004.

FRANÇA, I.S.X., SOUZA, A.J., BAPTISTA, R.S., BRITTO, V.R.S. Medicina popular: benefícios e malefícios das plantas medicinais.Rev. Bras. de Enferm. V. 61, n. 2, p. 201-8, Brasília, 2008.

HOEFFEL, J.L.M., GONÇALVES, N.M., FADINI, A.A.B., SEIXAS, S.R.C.

Conhecimento tradicional e uso de plantas medicinais nas APA'S Cantareiras/SP e Fernão Dias/MG. Rev. Vitas, ${ }^{\circ}{ }^{\circ}$ 1, 2008.

KLEIN, T., LONGHINI, R., BRUSCHI. M. L., MELLO, J.C.P. Fitoterápicos: um Mercado promissor. Rev. Ciên. Farmac. Básic. Aplicadas. V.3, n.30, p. 241-48, 2009.

MARTINS, E.R., CASTRO, D.M., CASTELLANI, D.C., DIAS, J.E. Plantas Medicianis. UFV, p. 220, Viçosa, 1998.

MORAIS, S.M. et al. Plantas medicinais usadas pelos índios Tapebas do Ceará. Rev. Bras. de Farmacog., v.11,n. 1, p. 21-35, 2001.

MORGAN, R. Enciclopédia das ervas e plantas medicinais : Doenças, Aplicações, 985 
Descrições e Propriedades. São Paulo- SP: Ed. Hermus, 1982.

NEWMAN, D.J., CRAGG, G.M. A fitoterapia no mundo atual. Nat. Prod., $p$ 70-461, 2007.

NICOLETTI, M.A., OLIVEIRA JUNIOR, M.A., BERTASSO, C.C., COPOROSSI, P.Y., OLIVEIRA, R. B., GODOY, S.A., COSTA, F.B. Plantas Tóxicas Conhecimento e Prevenção de Acidentes. Ed. Holos, p. 64, Ribeirão Preto - SP, 2003.

OLIVEIRA, R. B., GODOY, S.A., COSTA, F.B. Plantas Tóxicas Conhecimento e Prevenção de Acidentes. Ed. Holos, p. 64, Ribeirão Preto - SP, 2003.

PINILLOS, M.A., GÓMEZ, J., ELIZALDE, J. Intoxicación por alimentos, plantas e setas. Anales del Sistema Sanitário de Navarra, v. 16, n.1, p.243-263, Pamplona, 2003.

ROCHA, L.O., SOARES, M.M.S.R., CORREA, C.L. Análise da contaminação fúngica em amostras de Cássia acutifóliaDelile (sene) comercialização na cidade de Campinas, Brasil. Rev. Bras. Ciênc. Farmaceuticas., v.40, n ${ }^{\circ} 4$, p. 521527, 2004.

RIZZO, J.P., CAMPOS, I.P.P.. JAIME, M.V., MUNHOZ, G., MORGADO, W.F. Utilização de plantas medicinais nas cidades de Goiás e Pirenópolis, Estado de Goiás. Revista de Ciências Farmacêuticas, vol 20, nº 2, p. 431437, São Paulo, 1999.

SANTOS, J.F.L., AMOROZO, M.C.M., MING, L.C. Uso popular de plantas medicinais na comunidade de Vargem Grande, Município de Natividade da Serra São Paulo. Rev. Bras. Pl. Med., v.10, n.3, Botucatu, 2008.

\section{SCHENKEL, E.P. Cuidados com}

medicamentos. Ed. Medicina, $2^{\mathrm{a}}$ ed. Florianópolis - SC, 1996.
SILVA, B.D.C., OLIVEIRA, A.S., ARAÚJO, L.E., OLIVEIRA, L.M.S., ALMEIDA, L.M.M., COELHO, S.F.L.Estudo da atividade citotóxica de CleomespinosaJacp. Com o uso de Artemiasalina . In: Congresso Norte Nordeste de Pesquisa e Inovação, v.7, Palmas- TO, 2012.

SILVA, J.O., SOUZA, P.S. Levantamento etnobotânico das plantas medicinais utilizadas pela população da Vila Canaã região sudoeste - Goiânia, Goiás. Ciência Agrotécnica, v.32, p. 87-88. 2007.

SOUZA, S.A.M., MEIRA, M.R., FIGUEIREDO, L.S., MARTINS, E.R. Óleos essenciais: aspectos econômicos e sustentáveis. Enciclopédia Biosfera, v.6, n. 10, p.243-263, Goiânia, 2010.

VALE, N.B.A. Farmacobotânica, ainda tem lugar na moderna anestesiologia?Rev. Bras. Anestesiol, v.52, n. 3, p. 368-80, 2002.

VEIGA JUNIOR, V.F., PINTO, A.C., MACIEL, M.A.M. Plantas medicinais: cura segura? Química Nova, v.28, n. 3, p. 519528, São Paulo, 2005.

VEIGA JUNIOR, V.F. Estudo do consumo de plantas medicinais na Região Centro-Norte do Estado do Rio de Janeiro aceitação pelos profissionais de saúde e modo de uso pela população. Rev. Bras. deFarmacognósia, v. $18, \mathrm{n}^{\circ} 2,2008$.

VILA VERDE, G.M., PAULA, J.R., CARNEIRO, D.M. Levantamento etnobotânico das plantas medicinais do cerrado utilizadas pela população de Mossâmedes (GO).Rev. de Farmacog, v. 13, p. 64-66, 2003. 